\title{
openheart Novel cardiac pacemaker-based human model of periodic breathing to develop real-time, pre-emptive technology for carbon dioxide stabilisation
}

\author{
Resham Baruah, ${ }^{1}$ Alberto Giannoni, ${ }^{2}$ Keith Willson, ${ }^{1}$ Charlotte H Manisty, ${ }^{1,3}$ \\ Yoseph Mebrate, ${ }^{1,4}$ Andreas Kyriacou, ${ }^{1,4}$ Hemang Yadav, ${ }^{1,5}$ Beth Unsworth, ${ }^{1}$ \\ Richard Sutton, ${ }^{1}$ Jamil Mayet, ${ }^{1}$ Alun D Hughes, ${ }^{1,6}$ Darrel P Francis ${ }^{1}$
}

To cite: Baruah $\mathrm{R}$, Giannoni $\mathrm{A}$, Willson $\mathrm{K}$, et al. Novel cardiac pacemakerbased human model of periodic breathing to develop real-time, pre-emptive technology for carbon dioxide stabilisation. Open Heart 2014;1:e000055. doi:10.1136/openhrt-2014000055

Received 16 February 2014 Revised 23 May 2014 Accepted 15 July 2014

CrossMark

${ }^{1}$ International Centre for Circulatory Health, Imperial College Healthcare NHS Trust and Imperial College, London, UK

${ }^{2}$ Fondazione Gabriele Monasterio and Scuola Superiore Sant'Anna, Pisa, Italy

${ }^{3}$ The Heart Hospital, University College London, UK

${ }^{4}$ Royal Brompton and Harefield NHS

FoundationTrust, London, UK ${ }^{5}$ Mayo Clinic, Rochester, Minnesota USA

${ }^{6}$ Cardiovascular Physiology and Pharmacology, University College London, UK

Correspondence to Dr R Baruah; reshambaruah@gmail.com

\section{ABSTRACT}

Background: Constant flow and concentration $\mathrm{CO}_{2}$ has previously been efficacious in attenuating ventilatory oscillations in periodic breathing $(\mathrm{PB})$ where oscillations in $\mathrm{CO}_{2}$ drive ventilatory oscillations. However, it has the undesirable effect of increasing end-tidal $\mathrm{CO}_{2}$, and ventilation. We tested, in a model of $\mathrm{PB}$, a dynamic $\mathrm{CO}_{2}$ therapy that aims to attenuate pacemaker-induced ventilatory oscillations while minimising $\mathrm{CO}_{2}$ dose.

Methods: First, pacemakers were manipulated in 12 pacemaker recipients, 6 with heart failure (ejection fraction $(\mathrm{EF})=23.7 \pm 7.3 \%$ ) and 6 without heart failure, to experimentally induce PB. Second, we applied a real-time algorithm of pre-emptive dynamic exogenous $\mathrm{CO}_{2}$ administration, and tested different timings.

Results: We found that cardiac output alternation using pacemakers successfully induced $\mathrm{PB}$. Dynamic $\mathrm{CO}_{2}$ therapy, when delivered coincident with hyperventilation, attenuated $57 \%$ of the experimentally induced oscillations in end-tidal $\mathrm{CO}_{2}$ : $\mathrm{SD} /$ mean $0.06 \pm 0.01$ untreated versus $0.04 \pm 0.01$ with treatment $(p<0.0001)$ and $0.02 \pm 0.01$ in baseline nonmodified breathing. This translated to a $56 \%$ reduction in induced ventilatory oscillations: SD/mean $0.19 \pm 0.09$ untreated versus $0.14 \pm 0.06$ with treatment $(p=0.001)$ and $0.10 \pm 0.03$ at baseline. Of note, end-tidal $\mathrm{CO}_{2}$ did not significantly rise when dynamic $\mathrm{CO}_{2}$ was applied to the model $(4.84 \pm 0.47$ vs $4.91 \pm 0.45 \mathrm{kPa}$, $p=0.08)$. Furthermore, mean ventilation was also not significantly increased by dynamic $\mathrm{CO}_{2}$ compared with untreated $(7.8 \pm 1.2$ vs $8.4 \pm 1.2 \mathrm{~L} / \mathrm{min}$, $\mathrm{p}=0.17$ ).

Conclusions: Cardiac pacemaker manipulation can be used to induce PB experimentally. In this induced PB, delivering $\mathrm{CO}_{2}$ coincident with hyperventilation, ventilatory oscillations can be substantially attenuated without a significant increase in end-tidal $\mathrm{CO}_{2}$ or ventilation. Dynamic $\mathrm{CO}_{2}$ administration might be developed into a clinical treatment for PB.

Trial Registration number: ISRCTN29344450.

\section{KEY MESSAGES}

What is already known about this subject?

- Current treatments for central sleep apnoea, which may affect up to $50 \%$ of heart failure patients, have been marred by poor compliance possibly due to the fact that they obligate the use of tight fitting masks.

What does this study add?

- This study trials small doses of carefully-timed carbon dioxide which is delivered for short durations within each ventilatory cycle in a novel pacemaker model of periodic breathing.

How might this impact on clinical practice?

- It is hoped that this could be applied therapeutically for central sleep apneoa with a delivery system such as nasal cannulae which would avoid the use of tight-fitting masks.

\section{INTRODUCTION}

Periodic breathing (PB) is a waxing-waning ventilatory pattern, typically with a cycle length of $60 \mathrm{~s}$, generated by oscillations in respiratory gases $^{1}$ via an enhanced and delayed chemoreflex response. ${ }^{2-4}$ Common in heart failure (HF), ${ }^{5}{ }^{6} \mathrm{~PB}$ is associated with sleep disruption, adrenergic overactivation and increased mortality. ${ }^{7} 8$

Whether the physiological oscillations of PB contribute to the increased mortality is unknown, but it is plausible that by disrupting sleep they contribute to the fatigue that is characteristic of HF even in patients whose cardiac output at rest is not so low that fatigue would be expected.

A variety of techniques such as adaptive servoventilation (ASV) have been reported to improve sleep disordered breathing, left 
ventricular ejection fraction, exercise capacity and quality of life. ${ }^{9} 10$ Although long-term data are lacking, large randomised controlled trials are currently being conducted. Continuous positive airways pressure, an earlier and simpler technology, is uncomfortable for many patients. Its only large scale trial was unable to recruit more than two-thirds of the planned sample size and a further one-sixth of randomised patients withdrew from the trial; those remaining in the trial were using the therapy for less than $4 \mathrm{~h}$ per night after the first year. The trial did not show any mortality effect. ${ }^{11}$

Eliminating the requirement for tight fitting masks would permit a greater range of patients to find the therapy acceptable and might permit them to use it for longer during the night without discomfort. The key to eliminating the requirement for a tight-fit might be to not manipulate ventilation directly but rather use the patient's own chemoreflex to allow an intervention to be delivered in the form of $\mathrm{CO}_{2}$ and be translated into ventilation by the patient's own biology.

Prior attempts to use $\mathrm{CO}_{2}$ to alleviate $\mathrm{PB}$ have employed continuous, constant doses of exogenous $\mathrm{CO}_{2}$ throughout the $\mathrm{PB}$ cycle ('static' $\mathrm{CO}_{2}$ therapy), sometimes via the addition of dead-space, in order to raise the mean arterial $\mathrm{CO}_{2}$ level above the apnoeic threshold. While effective in reducing apnoeas, high doses of exogenous $\mathrm{CO}_{2}$ have the undesirable effects of potentiating the sympathetic nervous system and elevating mean ventilation. ${ }^{12-14}$ This is particularly hazardous in HF where the supply-demand balance is precarious and further increases in metabolic demand may be compromising. Static $\mathrm{CO}_{2}$ therapy, therefore, has not been implemented in routine clinical practice.

Previous work by our group suggests it may be possible to specifically target troughs in end-tidal $\mathrm{CO}_{2}$ using low-dose, carefully timed, brief administrations of $\mathrm{CO}_{2}$. This is analogous in many ways to ASV with therapy aimed at the current ventilatory instability. In dynamic $\mathrm{CO}_{2}$ administration, the aim is to attenuate or eliminate the troughs in $\mathrm{CO}_{2}$ that result from an enhanced chemoreflex gain and the hyperventilation phase of PB. In doing so, despite the intrinsic reflex being unchanged by the therapy, we aim to prevent the exaggerated response to what would have been the hypocapnia phase, namely the apnoea or hypopnoea that would then themselves have triggered a disproportionate rise in $\mathrm{CO}_{2}$. Wrongly timed 'dynamic' therapy, therefore, even with very small doses of $\mathrm{CO}_{2}$, has the potential to exacerbate ventilatory oscillations. Therefore, a system that allows prediction of the timing and size of troughs in end-tidal $\mathrm{CO}_{2}$ has been developed. ${ }^{15} 16$

Repetitive alternation of cardiac output using cardiac permanent pacemakers (PPMs) can engender oscillations in respiratory gases and ventilation, a process which could be used to model $\mathrm{PB}$, thereby facilitating therapeutic algorithm development. ${ }^{16}{ }^{17}$ The alternation of cardiac output from high to low every $30 \mathrm{~s}$ produces oscillations in ventilation with cycle length of $60 \mathrm{~s}$ that are not unlike the oscillations of spontaneous PB. ${ }^{17}$
Unlike spontaneous $\mathrm{PB}$ seen in $\mathrm{HF}$, in this model there is a continuous exogenous driver to oscillation, such that any improvement following intervention may be confidently attributed to the intervention, useful because the severity of spontaneous $\mathrm{PB}$ is variable and unpredictable. ${ }^{18}$ Moreover, this experimental model, rather than models of voluntarily simulated $\mathrm{PB}$, allows monitoring of the effect on ventilation. ${ }^{19-22}$ The model is not identical to spontaneous $\mathrm{PB}$, whose cycle time and amplitude are different between different patients and can fluctuate with time, although in our experiment the therapy algorithm does not have prior knowledge of the true cycle time or amplitude and has to deduce this from the ventilatory patterns being observed in real time. This goes some way to simulating the ultimate clinical application environment while retaining the advantages of experimental reproducibility of an underlying PB pattern that is relatively consistent.

In this study, we applied pacemaker manipulation to produce an experimental model of PB by alternating cardiac output. We then aimed to deliver $\mathrm{CO}_{2}$ using our pre-emptive $\mathrm{CO}_{2}$ delivery system. This required real-time prediction of when $\mathrm{CO}_{2}$ was likely to fall, and then exogenous $\mathrm{CO}_{2}$ to be delivered to fill the troughs in end-tidal $\mathrm{CO}_{2}$, thereby preventing the resultant hypoventilation. We aimed to stabilise ventilation, while minimising the amount of $\mathrm{CO}_{2}$ administered and the consequent undesirable increase in mean ventilation.

\section{METHODS \\ Subjects}

Of 21 patients screened, 3 were excluded for demonstrating PB at baseline, 5 because of heart rates $>80 \mathrm{bpm}$ at rest and 1 for significant lung disease. Twelve patients with PPM (4 with cardiac resynchronisation devices, 8 with dual or single chamber pacemakers; table 1) were finally recruited from outpatient cardiac services between 2007 and 2009. All implants were at least 3 months prior to recruitment. Six patients had echocardiographic (ejection fraction $<50 \%$ ), and clinical features of HF and six had normal systolic function.

Exclusion criteria were implantable cardiac defibrillators with antitachycardia therapy set at rates lower than $120 \mathrm{bpm}$, conditions precluding lying for $90 \mathrm{~min}$, recent decompensation, ventilatory disorders, end-stage renal failure and medication affecting ventilatory drive. Within patients with $\mathrm{HF}$, there was no evidence of daytime $\mathrm{PB}$ or exercise oscillatory ventilation but two patients demonstrated cardiac cachexia. The six patients without HF had pacemaker implantation for the following indications: atrioventricular (AV) node ablation, vasovagal syncope, first-degree heart block, sick sinus syndrome, complete heart block and sinus bradycardia.

On the study day, patients were monitored for $30 \mathrm{~min}$ while recumbent on a couch, in order to exclude spontaneous PB. All patients gave informed consent for the study, which was approved by local research ethics 


\begin{tabular}{lll} 
Table 1 Baseline characteristics & \\
\hline & $\begin{array}{l}\text { Heart } \\
\text { failure }\end{array}$ & $\begin{array}{l}\text { Non-heart } \\
\text { failure }\end{array}$ \\
\hline $\mathrm{n}$ & 6 & 6 \\
Age (years) & $72.5 \pm 9.3$ & $71.0 \pm 8.8$ \\
Male & 5 & 3 \\
Height (cm) & $171.3 \pm 8.3$ & $166.8 \pm 10.2$ \\
Weight (kg) & $77.0 \pm 10.6$ & $83.4 \pm 15.6$ \\
Ejection fraction (\%) & $23.7 \pm 7.3$ & $54.3 \pm 7.2$ \\
Heart rate (bpm) & $64 \pm 15$ & $55 \pm 3$ \\
Cardiac output (L/min) & $4.5 \pm 21$ & $7.6 \pm 2.8$ \\
End-tidal CO $(\mathrm{kPa})$ & $4.6 \pm 0.7$ & $5.1 \pm 0.2$ \\
Mean ventilation (L/min) & $8.1 \pm 1.8$ & $7.5 \pm 1.5$ \\
NYHA 2/3/4 & $2 / 3 / 1$ & \\
Aetiology & & \\
\multicolumn{1}{l}{ Ischaemic } & 4 & \\
Dilatative & 1 & \\
Valvular & 1 & \\
Alcoholic & 0 & \\
Treatment & & \\
Biventricular pacemaker & 4 & \\
ACE inhibitor/ARBs & 4 & \\
Beta-blockers & 3 & \\
Aldosterone antagonists & 4 & \\
Diuretics & 2 & \\
\hline ARBs, Angiotensi $/$ Receptor & & \\
\hline
\end{tabular}

ARBs, Angiotensin II Receptor Blockers; NYHA, New York Heart Association.

committee (05/Q0404/018). The investigation conformed to the principles outlined in the Declaration of Helsinki.

\section{Measurements}

Patients breathed through a calibrated pneumotachograph attached to a Multicap monitor (Datex Instumentarium, Helsinki, Finland) measuring ventilation and respiratory gases. An electrocardiogram signal was recorded using a Hewlett-Packard 78351A. Beat-by-beat blood pressure and cardiac output were measured non-invasively using a photoplethysmograph device (Finometer, Finapres Medical Systems, The Netherlands). ${ }^{23}$

\section{Data acquisition}

The data were sampled at $1000 \mathrm{~Hz}$ using a custom data acquisition system consisting of an analogue-to-digital card (DAQCard 6062E, National Instruments, Austin, Texas, USA) and a workstation running software written in Labview instrument control language (V.7.0, National Instruments). This system allowed data to be collected simultaneously from all the devices and later analysed off-line using software written in Matlab (Natick, Massachusetts, USA). ${ }^{16-18}$ Heart rate, blood pressure, cardiac output, end-tidal gas concentrations and ventilation were digitally interpolated and resampled to obtain signals at $1 \mathrm{~Hz}$ for subsequent analysis. ${ }^{13} 14$

\section{Pacemaker protocol}

Pacemaker reprogramming was performed via a pacemaker telemetry head positioned over the implanted pacemaker device. In all patients, the maximum possible safe change in cardiac output was elicited by changing heart rate, AV delay and pacing configuration where possible (eg, from right ventricular pacing to biventricular pacing). On average, the heart rate was changed from $59.5 \pm 10.2$ to $79.1 \pm 10.7 \mathrm{bpm}(\mathrm{p}<0.01)$. Cardiorespiratory variables were monitored while alternating the cardiac output as a step change, between low cardiac output and high cardiac output, every $30 \mathrm{~s}$ for at least five cycles of $60 \mathrm{~s}$ (untreated). Cardiac output was altered in exactly the same way for exactly the same duration during the dynamic $\mathrm{CO}_{2}$ therapy administrations.

\section{$\mathrm{CO}_{2}$ administration system}

Starting from a fixed concentration of $\mathrm{CO}_{2}$ in a nonpressurised reservoir, we delivered exogenous $\mathrm{CO}_{2}$ using a specially designed motorised valve, in any desired configuration using custom software (Matlab, Natick, Massachusetts, USA, ${ }^{13}{ }^{14}{ }^{16-18}$ ). This performed real-time analysis of ventilation and, using Fourier transformation, calculated the magnitude and the phase of ventilatory oscillations within the cycle at $1 \mathrm{~s}$ intervals during the experiment.

The motor drove a valve, which enabled the inspired gas mixture composition to be varied continuously to any desired value between atmospheric and the concentration of the $\mathrm{CO}_{2}$ cylinder. The administration algorithm was programmed to give $\mathrm{CO}_{2}$ doses dependent on the magnitude and phase of the current oscillation (figure 1).

The $\mathrm{CO}_{2}$ concentration was varied smoothly: it began from zero concentration, then rose smoothly to the peak of administration and then declined similarly to finish at zero again (figure 2). The peak concentration administered was automatically adjusted in real time to be proportional to the amplitude of the ventilatory oscillations, in order to prevent either over or under treatment $^{12}$ (figure 3). The dose required was recalculated every second based on the above principles and was translated into an electrical output to the motor. In the case of no ventilatory oscillations, no $\mathrm{CO}_{2}$ was delivered (figure 3).

\section{$\mathrm{CO}_{2}$ administration protocol}

During baseline cardiac output alternation (at least five $60 \mathrm{~s}$ cycles), no $\mathrm{CO}_{2}$ was administered. $\mathrm{CO}_{2}$ was then delivered at a phase that allowed peak alveolar concentration to be coincident with peak ventilation, for at least five $60 \mathrm{~s}$ cycles. We aimed to set the timing of delivery at the motor so that the peak concentration of inspired $\mathrm{CO}_{2}$ would be arriving at the alveolar level at approximately the peak of ventilation. In each subject, the time interval between motor movement and the increment in end-tidal $\mathrm{CO}_{2}$ during baseline breathing was measured and on average this was $9 \mathrm{~s}$. 

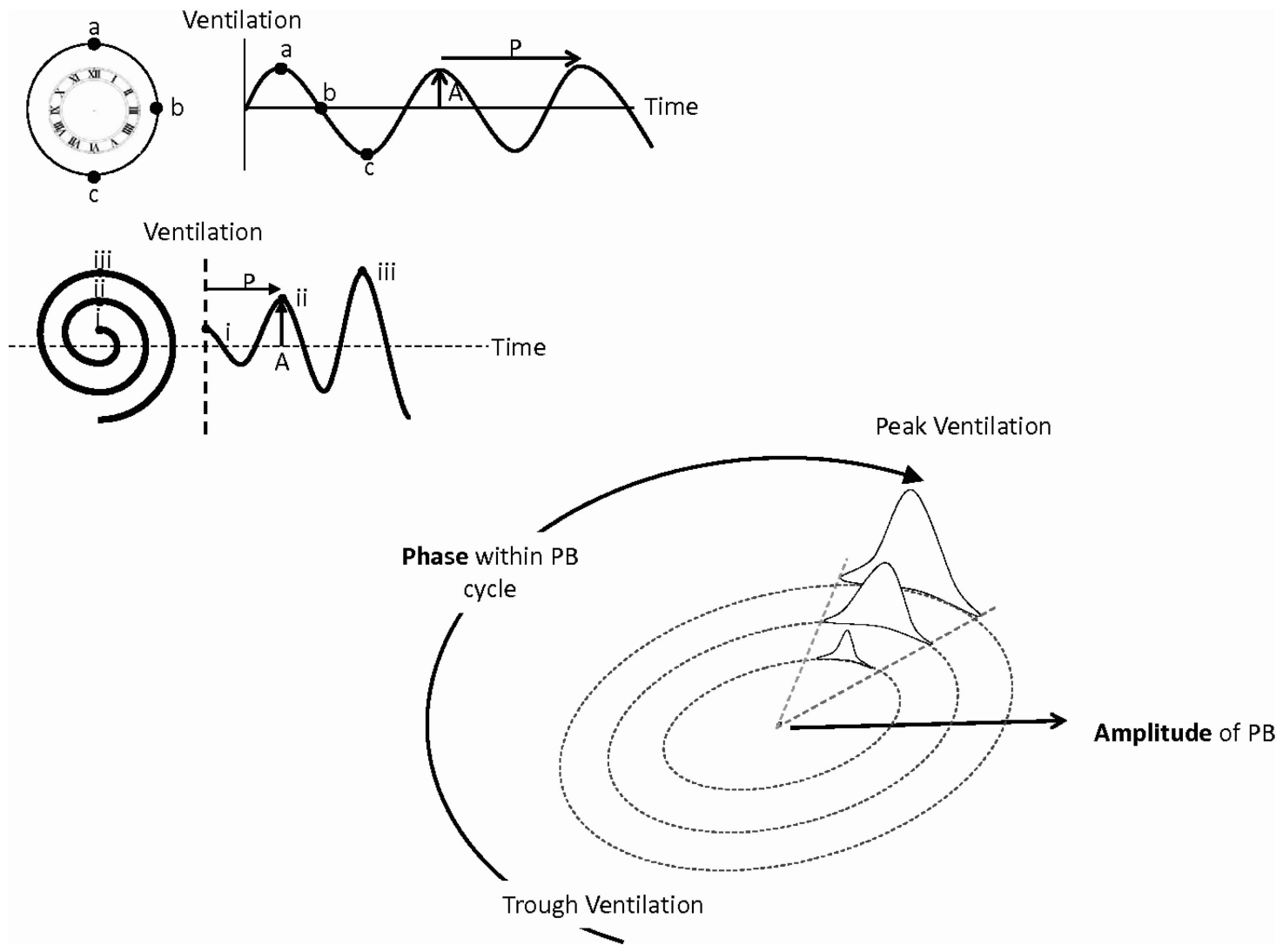

Figure 1 Top left: Each ventilatory cycle may be graphically represented as a clock with peak ventilation (a) at 12 o'clock, mid-ventilation (b) at either 3 o'clock or 9 o'clock (depending on whether ventilation is decreasing or increasing) and trough ventilation at 6 o'clock. Fourier analysis allows the amplitude $(A)$ and cycle length $(P)$ to be established. Low amplitude oscillations (i) are represented as nearer to the centre of the clock than medium (ii) or large (iii) oscillations. At any time, the current position within the PB cycle can, therefore, be appreciated by the position of the cursor. As ventilation stabilises the cursor moves closer towards the centre of the clock face. Bottom right: $\mathrm{CO}_{2}$ was delivered from 0 to peak concentration gradually and then falling back to 0 with a '1-cos' profile for a fraction of the PB cycle. Here dynamic $\mathrm{CO}_{2}$ is being delivered coincident with peak ventilation. Both the duration and peak dose of $\mathrm{CO}_{2}$ delivered are proportional to the size of the oscillations so the smaller oscillations receive a lower peak $\mathrm{CO}_{2}$ concentration for a shorter period of time (smallest sinusoidal administration) and the largest ventilatory oscillations receive both a longer administration and a higher peak $\mathrm{CO}_{2}$ concentration (largest sinusoidal profile).

In between interventions there was a 'washout period' where cardiac output continued to be alternated but no $\mathrm{CO}_{2}$ delivered. In a separate run, in order to assess the effect of phase on ventilation, $\mathrm{CO}_{2}$ was delivered so that the $\mathrm{CO}_{2}$ would arrive coincident at the alveolar level with trough ventilation, in antiphase to the initial therapeutic algorithm.

Figure 2 Schematic of laboratory set-up. $\mathrm{CO}_{2}$ and air are allowed to mix to any desired concentration using a computer-controlled motorised valve. This mixture is drawn in by the patient on inspiration and expired via a three-way valve. Inspired and expired $\mathrm{CO}_{2}$ concentrations and respiratory flow were measured and fed back to an online computer program to establish the position within the ventilatory cycle and to display this and the end-tidal $\mathrm{CO}_{2}$.

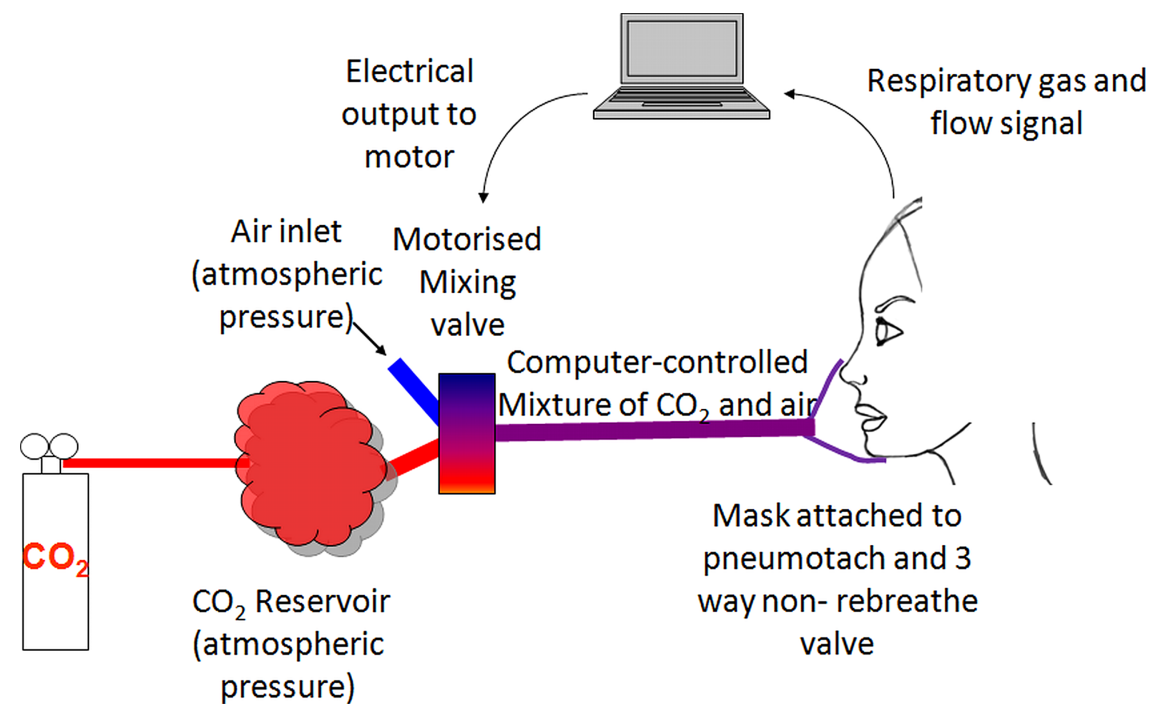




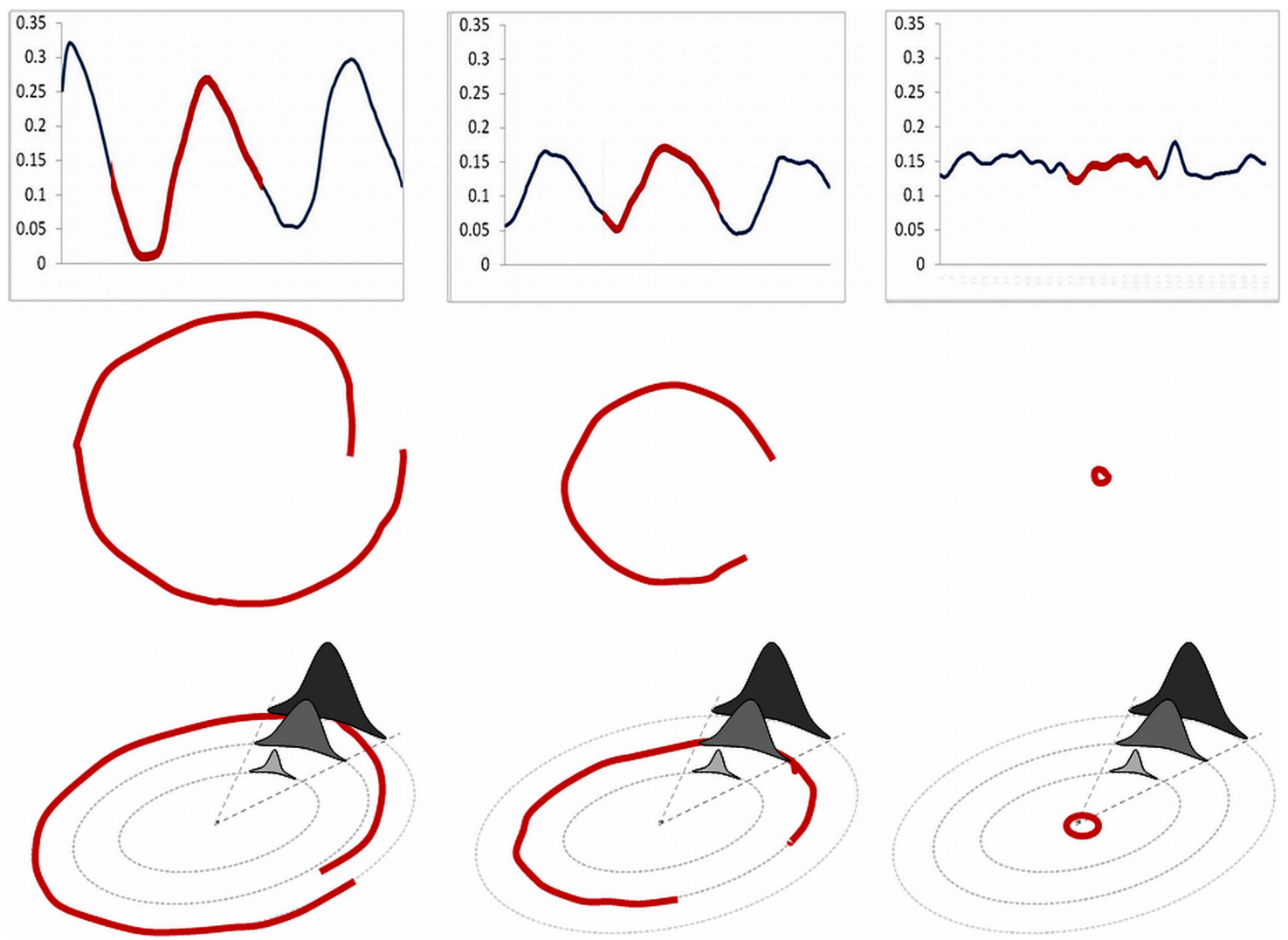

Figure 3 Upper left panel is the ventilation trace of a patient with spontaneous periodic breathing who has large ventilatory oscillations. Part of the trace (in red) has been translated to the middle left plot to demonstrate what would be seen in the lab in real time, where it progresses in a clockwise direction. This portion of the cycle starts in mid-cycle (at 3 o'clock). Ventilation then falls to its trough at 6 o'clock and then rises again to peak ventilation. As this second oscillation in the group of three is of lower amplitude than the preceding peak (blue in the top left panel) this part of the trace ends at mid-cycle closer to the centre of the circle than where it started. The bottom left hand panel shows how the treatment algorithm at peak dose would be applied. The patient would receive a high peak dose and a long duration of therapy (dark grey). The middle panel depicts moderate oscillations, from a different patient with spontaneous PB, with the red portion starting just before trough ventilation and continuing until just after the peak of ventilation (middle plot). This patient would receive the middle grey treatment algorithm (bottom, middle plot). The right hand panel is that of stable breathing in which no therapy would be given by the algorithm. In this case the cursor on screen would trace a path close to the centre.

\section{Data analysis}

The degree of oscillation of the measured respiratory variables (a marker of the severity of the simulated $\mathrm{PB}$ ) was measured using the coefficient of variation $(\mathrm{SD} / \mathrm{mean})$. This has the advantage of being dimensionless.

\section{Statistical analysis}

Continuous values are expressed as the mean \pm SD. Paired $t$ tests were performed to compare the effect of baseline alternations with the subsequent administration strategies within individuals. A value of $\mathrm{p}<0.05$ was considered statistically significant.

\section{RESULTS}

\section{Cardiac pacemaker modulation as a model of periodic breathing}

Heart rate was alternated on average by $59.5 \pm 10.2$ to $79.1 \pm 10.7 \mathrm{bpm}$. AV delay was alternated in all the patients with $\mathrm{HF}$ and four of the patients without HF by a mean of $108 \pm 55 \mathrm{~ms}$. Three of the four patients with biventricular pacemakers were also simultaneously alternated between biventricular pacing and right ventricular pacing. The resting cardiac output measured on the Finapres device was $6.9 \pm 3.6 \mathrm{~L} / \mathrm{min}$. During alternations between the two cardiac output states, the lower cardiac output state averaged $6.6 \pm 3.6 \mathrm{l} / \mathrm{min}$ and upper averaged $7.3 \pm 4.1 \mathrm{l} / \mathrm{min}$. The amplitude of fluctuation (defined as half the difference between upper and lower) was 2.46 $\pm 2.24 \mathrm{~L} / \mathrm{min}$ representing a change in cardiac output of $3.6-30 \%$ from baseline.

Following the pacemaker-induced cardiac output increase, the sequence of events was first an elevation in cardiac output, followed by a rise in end-tidal $\mathrm{CO}_{2}$ and then by a rise in ventilation (figure 4 ).

Alternation of cardiac output significantly increased the degree of oscillation in end-tidal $\mathrm{CO}_{2}$ by $182 \%$ compared to baseline ( $\mathrm{SD} /$ mean end-tidal $\mathrm{CO}_{2}$ from $0.02 \pm 0.01$ to $0.06 \pm 0.01, \mathrm{p}<0.0001)$. This translated to a $90 \%$ increase in 
Cardiac Output alternation alone

+ Dynamic $\mathrm{CO}_{2}$ coincident with peak ventilation
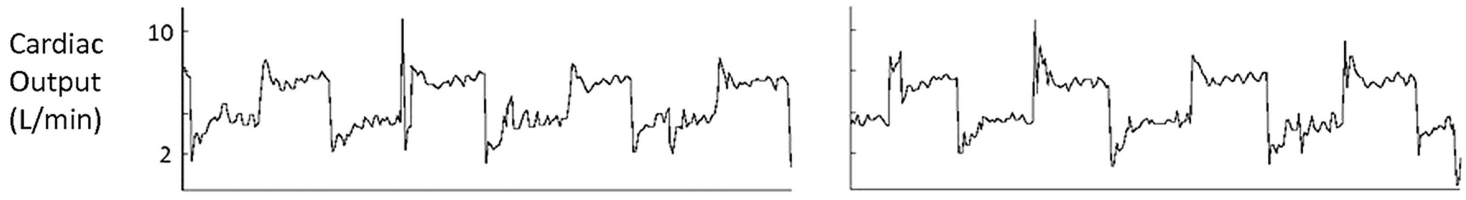

Valve

Position
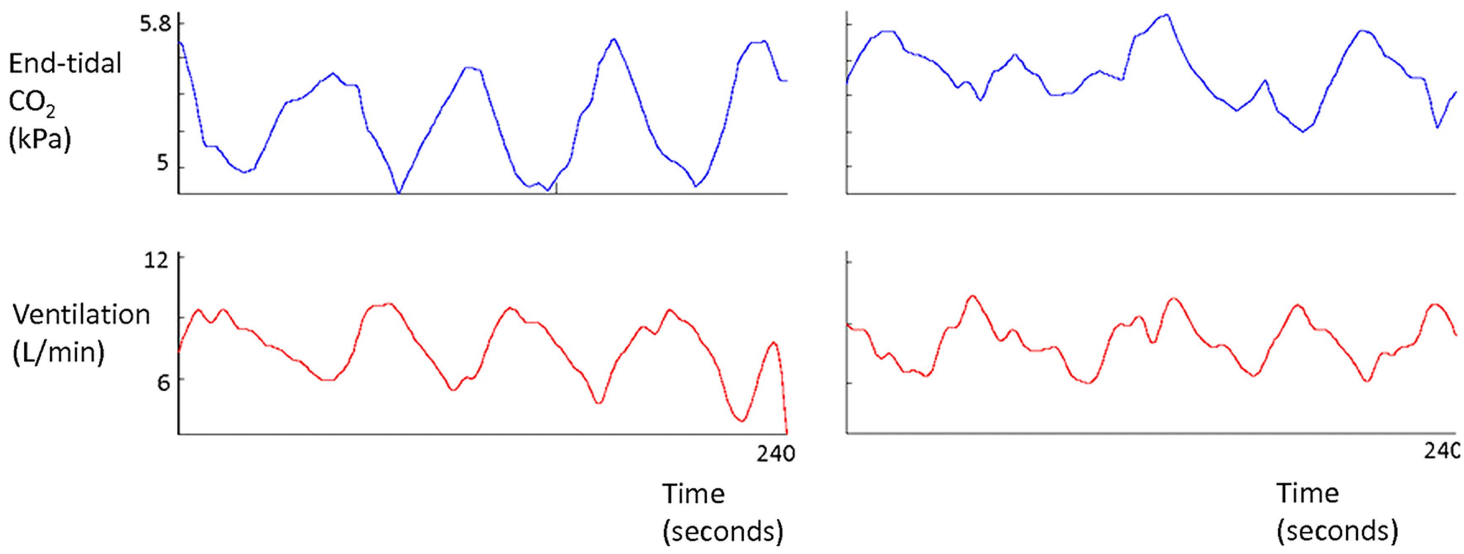

Figure 4 Left: Alternations in cardiac output (black) in one typical patient, every $60 \mathrm{~s}$ for three cycles, are followed by oscillations in end-tidal $\mathrm{CO}_{2}$ (blue) and ventilation (red), when no dynamic $\mathrm{CO}_{2}$ is applied. Right: Oscillations in end-tidal $\mathrm{CO}_{2}$ (blue) and ventilation (red), in the same patient, are attenuated when dynamic $\mathrm{CO}_{2}$ is delivered where the troughs in end-tidal $\mathrm{CO}_{2}$ are predicted (around peak ventilation). The motor valve position profile (green), which is proportional to the dose of $\mathrm{CO}_{2}$ delivered, varies in size and shape dependent on the amplitude and phase of the current ventilatory signal in real time. While ventilation is still oscillatory, the amplitude of the oscillations are diminished.
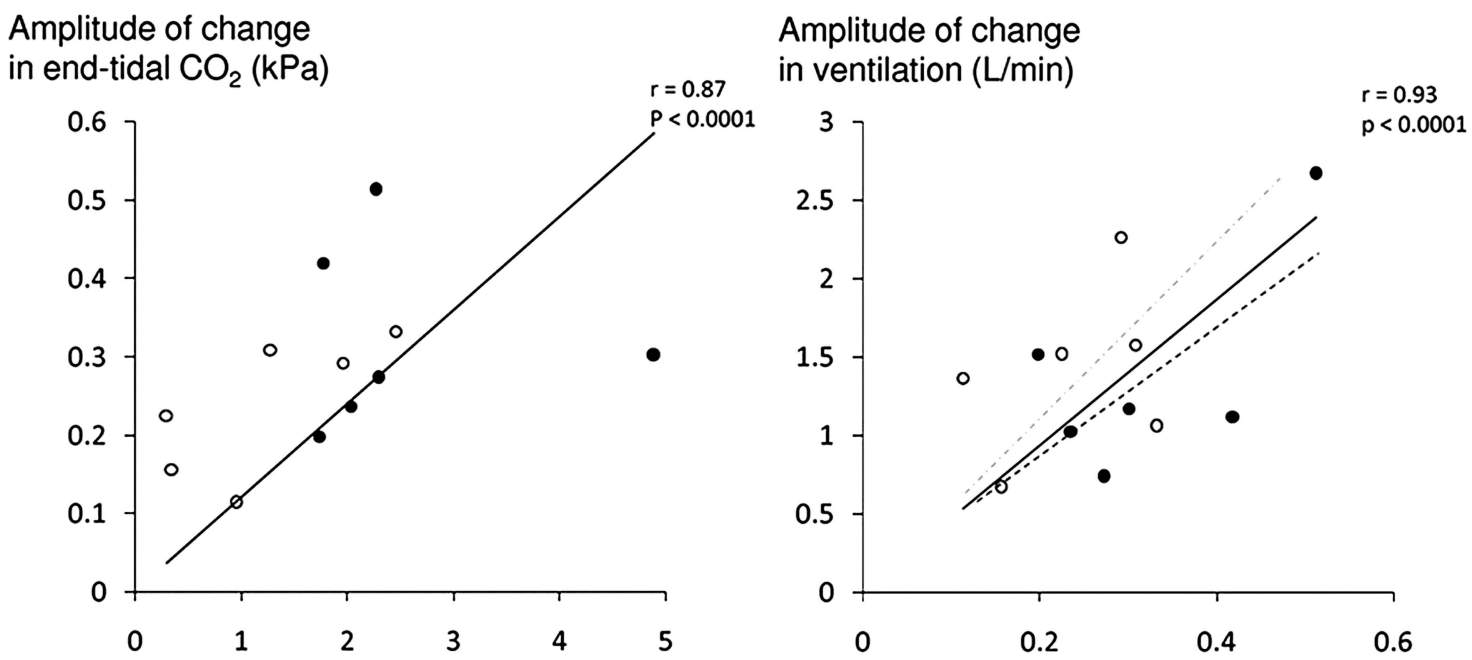

Amplitude of change in cardiac output (L/min)

Amplitude of change in end-tidal $\mathrm{CO}_{2}(\mathrm{kPa})$

Figure 5 The amplitude of oscillation of end-tidal $\mathrm{CO}_{2}$ correlates to the amplitude of oscillation of cardiac output for both patients with heart failure (no fill) and subjects with normal systolic function (solid black fill). The amplitude of ventilatory oscillation is closely correlated to the amplitude of oscillation of end-tidal $\mathrm{CO}_{2}$ with the regression line for heart failure patients in grey interrupted and for non-heart failure patients in black interrupted. Typically, for any given change in end-tidal $\mathrm{CO}_{2}$ there was a greater change in ventilation in the heart failure patients than the non-heart failure patients. 

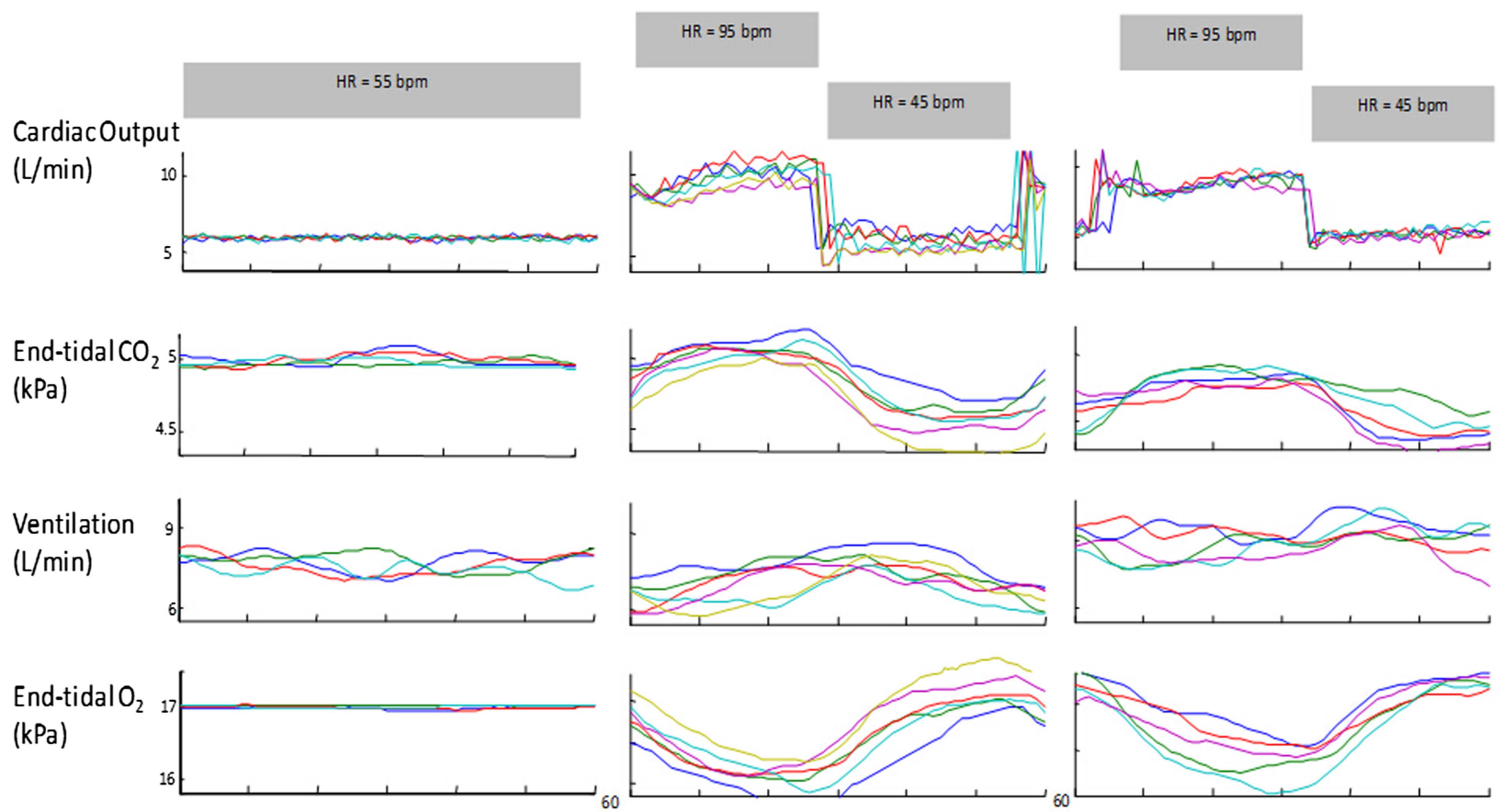

Figure 6 Raw data from one individual where each $60 \mathrm{~s}$ cycle has been time aligned. The far left hand panel is $240 \mathrm{~s}$ of baseline ventilation where no intervention has taken place. The middle panel is the data from cardiac output oscillations alone every thirty seconds resulting in oscillations in the respiratory gases and ventilation. The right hand panel shows five time-aligned cycles where dynamic $\mathrm{CO}_{2}$ therapy was applied coincident with peak ventilation while $60 \mathrm{~s}$ cardiac output alternation continued and the resultant amplitude of end-tidal $\mathrm{CO}_{2}$ and ventilatory oscillations were attenuated.

the degree of ventilatory oscillation (SD/mean from 0.10 \pm 0.03 to $0.19 \pm 0.09, \mathrm{p}=0.002$; figure 5 ). The resultant oscillations in end-tidal $\mathrm{CO}_{2}$ and ventilation were highly sinusoidal (fit-to-sine wave had $\mathrm{r}^{2}=0.95, \mathrm{p}<0.001$ and $\mathrm{r}^{2}=0.88$, $\mathrm{p}<0.001$ respectively, figures 4 and 6 ).

The amplitude of cardiac output change generated in patients without $\mathrm{HF}$ was greater than that generated by those with $\mathrm{HF}(3.68 \pm 2.58 \mathrm{~L} / \mathrm{min}$ vs $1.23 \pm 0.86 \mathrm{~L} / \mathrm{min}$, $\mathrm{p}=0.05$ ) and as a result the change in the end-tidal $\mathrm{CO}_{2}$ oscillations induced by the PPM was higher in the patients without $\mathrm{HF}(\Delta \mathrm{SD} /$ mean $=0.04 \pm 0.01$ vs 0.03 $\pm 0.01, \mathrm{p}=0.02$ ). However, there was no difference in the degree of ventilatory oscillation induced in patients with and without $\mathrm{HF}(\Delta \mathrm{SD} / \mathrm{Mean}=0.08 \pm 0.05$ vs $0.10 \pm 0.10$, $\mathrm{p}=0.73$ ). This may be explained by the trend towards the ratio between the rise in ventilation and that in end-tidal $\mathrm{CO}_{2}$ (a form of chemoreflex gain assessment), being higher in the patients with $\mathrm{HF}$ than without (608.4 \pm 233.5 vs $385.9 \pm 118.7 \mathrm{~L} / \mathrm{min} / \mathrm{kPa}, \mathrm{p}=0.06$, figure 5$)$. Similarly, there was a non-significant trend towards the time taken between the peak in end-tidal $\mathrm{CO}_{2}$ and the peak ventilation (a measure of chemoreflex delay), being longer for patients with HF than in without (22.4 \pm 3.1 vs $16.9 \pm 6.6 \mathrm{~s}, \mathrm{p}=0.09$ ).

\section{Dynamic $\mathrm{CO}_{2}$ delivery coincident with peak ventilation}

In order for the supplemental inspired $\mathrm{CO}_{2}$ to arrive at the alveolar level coincident with the peak of ventilation, the motor had to begin its movement substantially in advance of the peak of ventilation. The algorithm did not require sensing of any particular level of ventilation but rather was able to project into the future the curve of oscillation of ventilation. What was programmed into the algorithm was the time within the PB cycle at which the valve should have the greatest aperture. The average delay between $\mathrm{CO}_{2}$ being delivered and the inspired $\mathrm{CO}_{2}$ rising was $8.8 \pm 2.5 \mathrm{~s}$. With dynamic $\mathrm{CO}_{2}$ administration that was timed to be coincident with peak ventilation, the size of the variation in end-tidal $\mathrm{CO}_{2}$ fell from the untreated level of $0.06 \pm 0.01$ to $0.04 \pm 0.01, p=0.001$, which is $57 \%$ of the way to the value in baseline nonmodulated breathing of $0.02 \pm 0.01$. Dynamic $\mathrm{CO}_{2}$ achieved a $56 \%$ reduction in the variability of ventilation from the experimentally induced oscillations from 0.19 \pm 0.09 to $0.14 \pm 0.06, \mathrm{p}=0.001$, where baseline breathing alone had oscillations of $0.10 \pm 0.03$ (figures 4, 6 and 7).

There was a trend towards increased mean end-tidal $\mathrm{CO}_{2}$ following application of the dynamic $\mathrm{CO}_{2}(4.91 \pm$ 0.45 vs $4.84 \pm 0.47 \mathrm{kPa}, \mathrm{p}=0.08)$ and mean ventilation compared to the pacemaker-induced $\mathrm{PB}(8.4 \pm 1.2$ vs 7.8 $\pm 1.2 \mathrm{~L} / \mathrm{min}, \mathrm{p}=0.17$; figures 4,7 and 8 ).

\section{Dynamic $\mathrm{CO}_{2}$ delivery to coincide with trough ventilation}

In contrast, delivering dynamic $\mathrm{CO}_{2}$ therapy just before trough ventilation (ie, at the opposite phase to that which is predicted to be helpful) failed to attenuate 

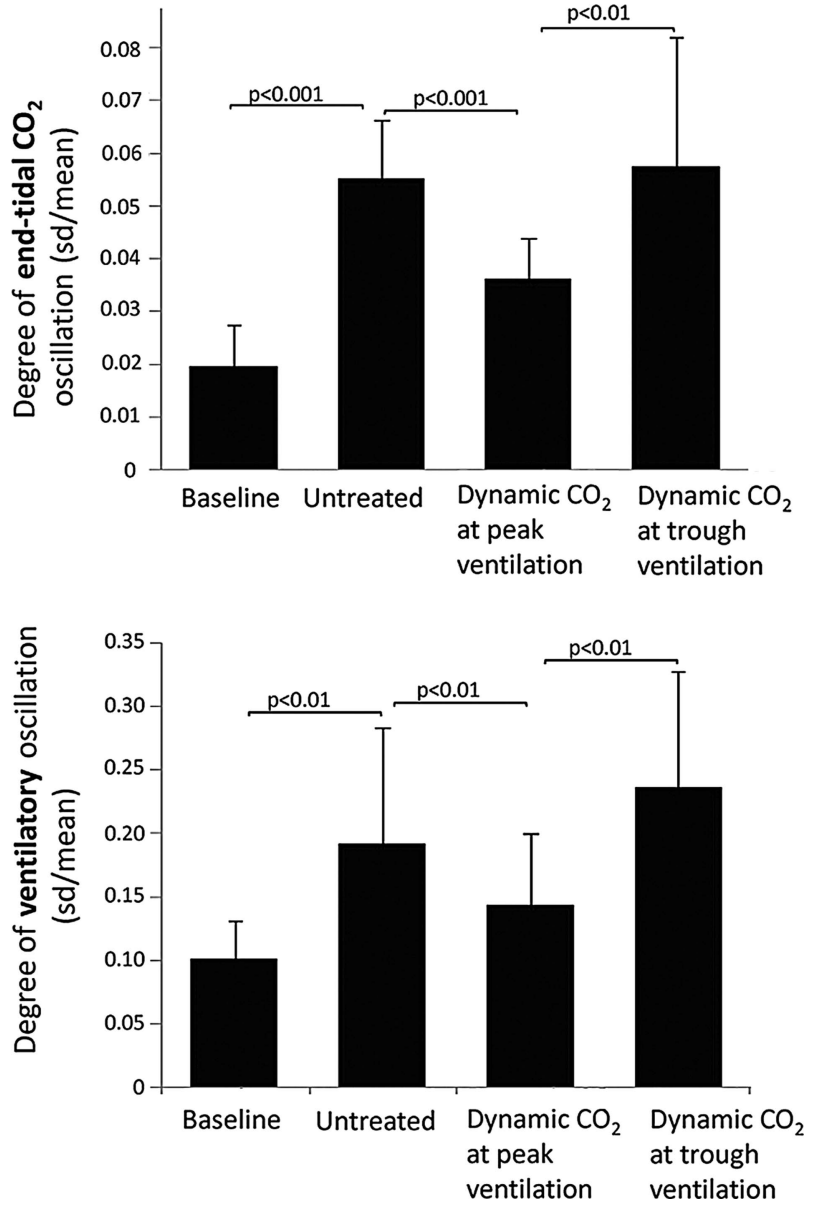

Figure 7 By alternating cardiac output via pacemakers labelled 'Untreated', there was a $182 \%$ increase in end-tidal $\mathrm{CO}_{2}$ oscillations compared to stable breathing without intervention labelled 'Baseline'. When dynamic $\mathrm{CO}_{2}$ was applied to the experimentally induced oscillations coincident with peak ventilation, this abolished $57 \%$ of the induced oscillations. When dynamic $\mathrm{CO}_{2}$ was applied $180^{\circ}$ after peak ventilation there was no significant difference to pacemaker-induced oscillations alone. Degree of ventilatory oscillation during stable breathing 'Baseline', with alternation of cardiac output using pacemakers 'Untreated', when dynamic $\mathrm{CO}_{2}$ is applied at peak ventilation and following application of dynamic $\mathrm{CO}_{2}$ coincident with trough ventilation.

both the degree of oscillation of end-tidal $\mathrm{CO}_{2}(0.06$ \pm 0.02 at antiphase vs $0.06 \pm 0.01$, untreated, $\mathrm{p}=0.75$ ) or ventilation $(0.024 \pm 0.09$ at antiphase vs $0.19 \pm 0.09$ untreated, $\mathrm{p}=0.17$, figure 7 , table 2 ).

\section{DISCUSSION}

This study demonstrates that it is possible to produce a human model of PB using cardiac pacemaker manipulation both in patients with preserved systolic function and in patients with HF (figures 4, 5 and 6). The enhanced chemoreflex gain that is seen in patients with $\mathrm{HF}$ increases the ability of manipulations of cardiac output to affect ventilation. We were able to apply this model of $\mathrm{PB}$ to develop a potential therapeutic approach; to

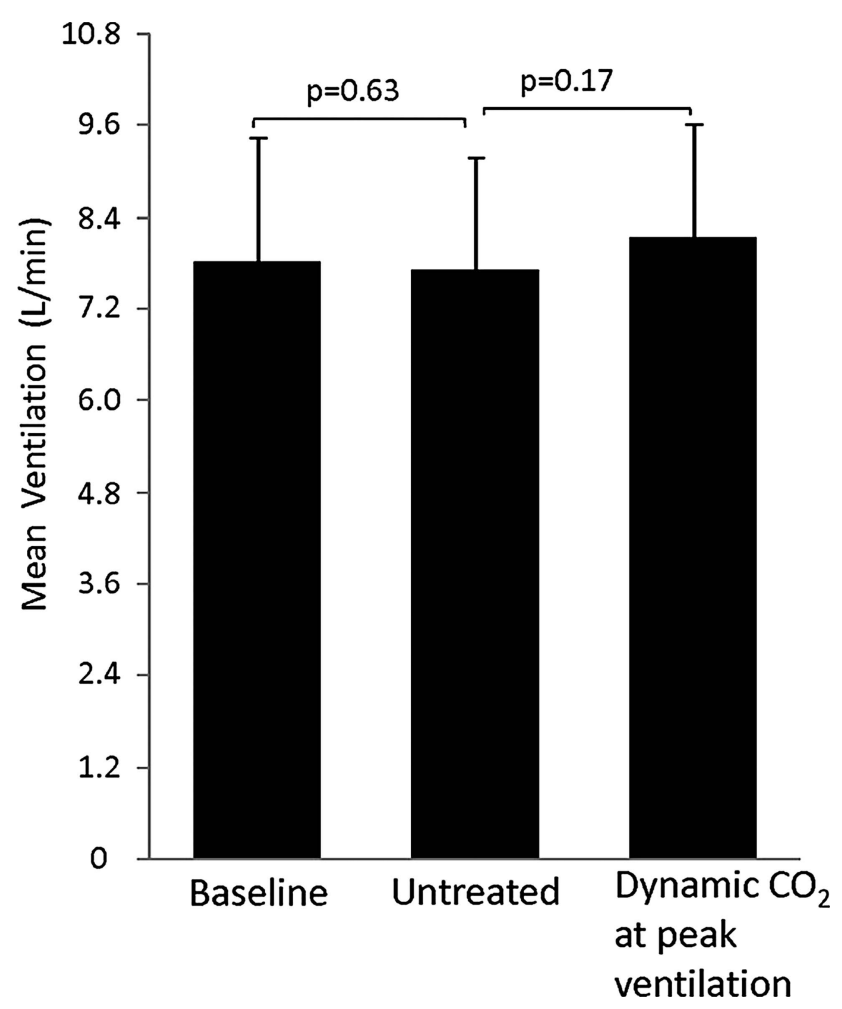

Figure 8 Dynamic $\mathrm{CO}_{2}$ administration, which successfully attenuated $57 \%$ of the induced oscillations in end-tidal $\mathrm{CO}_{2}$, was not associated overall with a significant increase in mean ventilation compared with untreated experimentally induced pacemaker oscillations alone.

deliver small, graded amounts of $\mathrm{CO}_{2}$ carefully timed within the PB cycle. ${ }^{12}$ This improved stability and minimised the increment in end-tidal levels of $\mathrm{CO}_{2}$ and mean ventilation whose adverse effects might have prevented a simpler static $\mathrm{CO}_{2}$ regime from being adopted as a therapy.

\section{A dynamic solution for a dynamic problem?}

Ventilation is tightly controlled by negative feedback whereby a rise in arterial $\mathrm{CO}_{2}$ elicits the reflex physiological response of increased ventilation which tends to reduce arterial $\mathrm{CO}_{2}$. In conditions such as $\mathrm{PB}$ where this chemoreflex is exaggerated and delayed, ${ }^{24}$ this reflex response actually generates oscillations in end-tidal $\mathrm{CO}_{2}$ and ventilation. ${ }^{25}$

Previously, low arterial $\mathrm{CO}_{2}$ concentrations seen in $\mathrm{PB}$ and $\mathrm{HF}$ have been targeted using static $\mathrm{CO}_{2}$ (either by continuous supplementary $\mathrm{CO}_{2}$ or by adding deadspace). While often successful in stabilising ventilation, this is at the clinically prohibitive cost of increased mean ventilation. $^{12}$

In the present study, we tested a dynamic administration algorithm. Rather than delivering $\mathrm{CO}_{2}$ statically, this gave small finely adjusted concentrations of $\mathrm{CO}_{2}$ for small parts of the PB cycle aiming to specifically fill in the troughs of $\mathrm{CO}_{2}$ produced by hyperventilation rather than to raise the overall $\mathrm{CO}_{2}$ level. ${ }^{12}$ 
Table 2 Results of dynamic $\mathrm{CO}_{2}$ administration on end-tidal $\mathrm{CO}_{2}$ and ventilation

\begin{tabular}{|c|c|c|c|c|c|c|c|c|}
\hline & Mean (kPa) & & SD/mean & & Mean (L/min) & & SD/mean & \\
\hline Baseline & $4.83 \pm 0.58$ & & $0.02 \pm 0.01$ & & $7.8 \pm 1.8$ & & $0.10 \pm 0.03$ & \\
\hline Untreated & $4.84 \pm 0.47$ & & $0.06 \pm 0.01$ & & $7.8 \pm 1.2$ & & $0.19 \pm 0.09$ & \\
\hline$p$ vs baseline & & 0.90 & & $<0.001$ & & 0.6 & & $<0.01$ \\
\hline Predicted optimum & $4.90 \pm 0.45$ & & $0.04 \pm 0.01$ & & $8.4 \pm 1.2$ & & $0.14 \pm 0.06$ & \\
\hline p vs baseline & & 0.40 & & $<0.001$ & & 0.5 & & $<0.01$ \\
\hline$p$ vs untreated & & 0.1 & & $<0.001$ & & 0.2 & & $<0.01$ \\
\hline Antiphase to predicted optimum & $4.85 \pm 0.43$ & & $0.06 \pm 0.02$ & & $7.2 \pm 1.8$ & & $0.24 \pm 0.09$ & \\
\hline p vs baseline & & 0.5 & & 0.001 & & 0.2 & & $<0.001$ \\
\hline$p$ vs untreated & & 0.2 & & 0.8 & & 0.30 & & 0.2 \\
\hline p vs optimal & & 0.2 & & 0 & & 0.1 & & $<0.01$ \\
\hline
\end{tabular}

Degree of oscillation and mean end-tidal $\mathrm{CO}_{2}$ and ventilation when the patients were quietly observed at rest 'Baseline', cardiac output was alternated using a pacemaker to induce oscillations 'Untreated', dynamic $\mathrm{CO}_{2}$ was applied to the induced oscillations coincident with peak ventilation 'Predicted Optimal', and dynamic $\mathrm{CO}_{2}$ was applied to the induced oscillations coincident with trough ventilation 'Antiphase to predicted optimum'.

\section{Why do we need a pacemaker model of periodic breathing?}

In clinical practice, $\mathrm{PB}$ and central sleep apnoea typically appear and disappear unpredictably during a recording, ${ }^{152627}$ presenting a problem while developing interventions where numerous aspects of the algorithm could, in principle, be adjusted. For example, in dynamic therapy, the timing of $\mathrm{CO}_{2}$ delivery is likely to be important, and a large number of variants of timing are possible. ${ }^{12}$ If each conceivable variant of timing had to undergo a whole night of testing in numerous patients, it would take an impractically long time to make progress. Likewise, the maximal concentration of $\mathrm{CO}_{2}$ and the profile of administration within the PB cycle are further aspects of the therapeutic algorithm that require investigation. If a grid of combinations of even these three aspects were to be tested, it would be even more impractical.

The purpose of our experimentally inducible PB is to allow ventilatory oscillations to be generated at will in patients, with a predictable and consistent pattern. ${ }^{13} 14$

\section{Pre-emptive predictive $\mathrm{CO}_{2}$ delivery: from theory to practice}

The findings of this study are consistent with other findings by our group that dynamic $\mathrm{CO}_{2}$ therapy may stabilise ventilatory oscillations, but only if the timing and dose used are correct. ${ }^{12} 16$ These studies identified that if dynamic $\mathrm{CO}_{2}$ was correctly timed to be coincident with peak ventilation, it had the potential to significantly attenuate the oscillations, but if $\mathrm{CO}_{2}$ delivery was incorrectly timed (arriving during the hypoventilation phase), it could even make breathing more unstable. ${ }^{12} 16$

\section{Potential clinical impact}

Static $\mathrm{CO}_{2}$ is not clinically utilised due to the undesirable consequences of large doses of $\mathrm{CO}_{2} \cdot{ }^{28}$ Dynamic $\mathrm{CO}_{2}$ therapy achieved significant amelioration of the induced PB but without increasing mean ventilation (figure 8). This is consistent with the work by our group, which suggests that dynamic $\mathrm{CO}_{2}$ administration can stabilise oscillatory ventilation without the cost of a statistically significant increase in mean ventilation. It is likely that there was in fact a small increment in this study, as some supplemental inspired $\mathrm{CO}_{2}$ was given. If we had used higher concentrations, the effect on $\mathrm{CO}_{2}$ and ventilation would have been proportionately larger. However, it is unlikely that administered over a short part of the $\mathrm{PB}$ cycle and in varying concentrations, it would amount to delivering the same amount of $\mathrm{CO}_{2}$ as is delivered with static $\mathrm{CO}_{2}$ therapy. The effect of static $\mathrm{CO}_{2}$ therapy on ventilation is not often explicitly stated but can be calculated from the equation below:

$$
\Delta \mathrm{V}=\frac{\mathrm{VCO}_{2}}{\Delta\left(\mathrm{FetCO}_{2}-\mathrm{FiCO}_{2}\right)}
$$

where $\Delta \mathrm{V}$ is the change in alveolar ventilation, $\mathrm{VcO}_{2}$ a constant volume of body production of $\mathrm{CO}_{2}$, $\Delta\left(\mathrm{FetCO}_{2}-\mathrm{FiCO}_{2}\right)$ is the change in difference of endtidal $\mathrm{CO}_{2}$ to inspired $\mathrm{CO}_{2}$.

This equation can also be used to calculate the rise in mean ventilation that occurred in the previous published clinical studies using both static $\mathrm{CO}_{2}$ delivery and the addition of even very small amounts of dead space, which increased ventilation ranges from $24 \%$ to $96 \% .{ }^{12}$ This degree of increase in ventilation imposes large increments in the patients' metabolic requirements, which may be particularly precariously balanced in $\mathrm{HF}$, in addition to the $\mathrm{CO}_{2}$-induced potentiation of the sympathetic nervous system and sleep disruption. ${ }^{24}$ However, the increment with dynamic $\mathrm{CO}_{2}$ is likely much smaller than this, by virtue of the fact that the overall dose of $\mathrm{CO}_{2}$ administered is so very much smaller. It is not known whether the efficacy of dynamic $\mathrm{CO}_{2}$ on attenuating ventilatory oscillations would be greater in spontaneous PB than it is in driven PB. In spontaneous PB each cycle of $\mathrm{PB}$ is the driver for the next. If the therapy attenuates one cycle, then this action may have an 
indirect effect of attenuating the second cycle, separate from the direct therapeutic effect on the second cycle.

Another advantage of a dynamic therapeutic approach to a physiological problem such as PB is that it would be automatically titrated to severity such that if breathing stabilises, therapy ceases.

This study also incidentally raises the possibility that cardiac output modulation by a pacemaker could be used therapeutically to counteract oscillation rather than to induce it.

\section{Study limitations}

This study only verifies that a PB pattern can be elicited at will and that this experimentally induced $\mathrm{PB}$ can be used to develop and refine dynamic $\mathrm{CO}_{2}$ algorithms. This is a proof-of-concept study and does not aim to assess the effect of this algorithm on spontaneous $\mathrm{PB}$ and, as a non-apnoeic model of $\mathrm{PB}$, may not mimic true apnoeic PB. In this study, the experimental PB was not completely eliminated but it was attenuated by about one half. This may be because of the limitations of dynamic $\mathrm{CO}_{2}$ delivery in attenuating ventilatory oscillations, or it may be because the driver to physiological oscillations (changes in cardiac output) was unremitting. If such a technology were developed and applied to spontaneous $\mathrm{PB}$, the degree of efficacy might be more or less than this, which can only be found from future randomised prospective trials. In addition, it is possible that even the level of decrease seen here may be sufficient to break the vicious circle that gives rise to PB.

This study was designed to detect mean effects across the entire group and, therefore, any comparisons between patients with and without HF are likely to be underpowered. One example is that there was only a trend towards longer chemoreflex delays in patients with HF compared with without HF, whereas previous studies have consistently reported this difference to be statistically significant. ${ }^{4}$

A facemask and pneumotach were used to unambiguously quantify the concentration of $\mathrm{CO}_{2}$ being inspired and the end-tidal $\mathrm{CO}_{2}$. Although such masks are poorly tolerated in normal clinical practice, ${ }^{29}$ and they necessitate an element of dead space, dynamic $\mathrm{CO}_{2}$ administration need not be dependent on tightly fitting masks.

This particular study did not address whether static $\mathrm{CO}_{2}$ therapy would have been efficacious in reducing the experimentally induced oscillations, and at what cost to mean ventilation, in this novel model of PB. Future studies might benefit from incorporation from a static $\mathrm{CO}_{2}$ arm that is either matched to the dynamic $\mathrm{CO}_{2}$ arm, either in terms of the average amount of $\mathrm{CO}_{2}$ delivered over the cycle, or in terms of efficacy on ventilatory stability. The latter option might be complex because it would require the dynamic therapy to always be done first and then a variety of static $\mathrm{CO}_{2}$ concentrations to be tested in order for the equivalent one to be identified.

\section{CONCLUSION}

We describe a method of inducing PB at will in subjects with pacemakers that permits evaluation of the impact of proposed therapies on ventilatory patterns in a controlled manner. Further, we developed a real-time preemptive $\mathrm{CO}_{2}$ stabilisation system that relies only on ventilatory monitoring. ${ }^{12}{ }^{16}$ Such a system allowed successful prediction of where end-tidal $\mathrm{CO}_{2}$ was likely to fall, and by delivering controlled doses of inhaled $\mathrm{CO}_{2}$ at this time, it was possible to substantially attenuate both oscillations in end-tidal $\mathrm{CO}_{2}$ oscillations and ventilation. Critically, ventilatory oscillations were attenuated without increasing either mean end-tidal $\mathrm{CO}_{2}$ or mean ventilation, making pre-emptive dynamic $\mathrm{CO}_{2}$ therapy a potentially attractive therapeutic intervention in conditions such as $\mathrm{PB}$ in $\mathrm{HF}$.

Contributors The study was conceived of and planned by RB, KW, CM, YB, RS, JM, ADH and DPF. RB, AG, KW, YM, AK, HY and BU performed the study. RB, AG, AK, HY, JM, ADH and DPF analysed the data. All authors contributed to the writing of the manuscript and have approved the final version. All authors agree that they are accountable for all aspects of the work in ensuring that questions related to the accuracy or integrity of any part of the work are appropriately investigated and resolved. DPF is the guarantor.

Funding RB (PG/07/065), DPF (FS/04/079), AK (FS/08/027/24763) and BU (PG/07/066) were supported by the British Heart Foundation. AG was supported by a European Society Cardiology research grant. KW received support from the Foundation for Circulatory Health (ICCH/05/5004), and CM was supported by a Welcome Trust Fellowship (077049/Z/05/Z). The authors are grateful for support from the NIHR Biomedical Research Centre funding scheme.

Competing interests None.

Patient consent Obtained.

Ethics approval Royal Brompton and Harefield NHS Trust ethics committtee.

Provenance and peer review Not commissioned; externally peer reviewed.

Data sharing statement No additional data are available.

Open Access This is an Open Access article distributed in accordance with the terms of the Creative Commons Attribution (CC BY 3.0) license, which permits others to distribute, remix, adapt and build upon this work, for commercial use, provided the original work is properly cited. See: http:// creativecommons.org/licenses/by/3.0/

\section{REFERENCES}

1. Ponikowski $P$, Anker SD, Chua TP, et al. Oscillatory breathing patterns during wakefulness in patients with chronic heart failure: clinical implications and role of augmented peripheral chemosensitivity. Circulation 1999;100:2418-24.

2. Solin P, Roebuck T, Johns DP, et al. Peripheral and central ventilatory responses in central sleep apnea with and without congestive heart failure. Am J Respir Crit Med 2000;162:2194-200.

3. Javaheri S. A mechanism of central sleep apnea in patients with heart failure. N Engl J Med 1999;341:949-54.

4. Francis DP, Willson K, Davies LC, et al. Quantitative general theory for periodic breathing in chronic heart failure and its clinical implications. Circulation 2000;102:2214-21.

5. Javaheri S, Parker TJ, Liming JD, et al. Sleep apnea in 81 ambulatory male patients with stable heart failure: types and their prevalences, consequences, and presentations. Circulation 1998;97:2154-9.

6. Mortara A, Sleight P, Pinna GD, et al. Abnormal awake respiratory patterns are common in chronic heart failure and may prevent evaluation of autonomic tone by measures of heart rate variability. Circulation 1997;96:246-52. 
7. Lanfranchi PA, Braghiroli A, Bosimini E, et al. Prognostic value of nocturnal Cheyne-Stokes respiration in chronic heart failure. Circulation 1999;99:1435-40.

8. Hastings PC, Vazir A, O'Driscoll DM, et al. Symptom burden of sleep-disordered breathing in mild-to-moderate congestive heart failure patients. Eur Respir J 2006;27:748-55.

9. Pepperell JC, Maskell NA, Jones DR, et al. A randomized controlled trial of adaptive ventilation for Cheyne-Stokes breathing in heart failure. Am J Respir Crit Care Med 2003;168:1109-14.

10. Oldenburg O, Bitter T, Lehmann R, et al. Adaptive servoventilation improves cardiac function and respiratory stability. Clin Res Cardiol 2011:100:107-15.

11. Arzt M, Floras JS, Logan AG, et al. Suppression of central sleep apnea by continuous positive airway pressure and transplant-free survival in heart failure: a post hoc analysis of the Canadian Continuous Positive Airway Pressure for Patients with Central Sleep Apnea and Heart Failure Trial (CANPAP). Circulation 2007;115:3173-80.

12. Steens RD, Millar TW, Su X, et al. Effect of inhaled $3 \% \mathrm{CO}_{2}$ on Cheyne-Stokes respiration in congestive heart failure. Sleep 1994;17:61-8.

13. Lorenzi-Filho G, Rankin F, Bies I, et al. Effects of inhaled carbon dioxide and oxygen on cheyne-stokes respiration in patients with heart failure. Am J Respir Crit Care Med 1999;159:1490-8.

14. Andreas S, Weidel K, Hagenah G, et al. Treatment of Cheyne-Stokes respiration with nasal oxygen and carbon dioxide. Eur Respir J 1998;12:414-19.

15. Mebrate $\mathrm{Y}$, Willson $\mathrm{K}$, Manisty $\mathrm{CH}$, et al. Dynamic $\mathrm{CO}_{2}$ therapy in periodic breathing - a modelling study to determine optimal timing and dosage regimes. J Appl Physiol 2009;107:696-706.

16. Manisty $\mathrm{CH}$, Willson K, Davies JE, et al. Induction of oscillatory ventilation pattern using dynamic modulation of heart rate through a pacemaker. Am J Physiol Regul Integr Comp Physiol 2008;295: R219-27.

17. Baruah R, Manisty $\mathrm{CH}$, Giannoni A, et al. Novel use of cardiac pacemakers in heart failure to dynamically manipulate the respiratory system through algorithmic changes in cardiac output. Circ Heart Fail 2009;2:166-74.

18. Caples SM, Wolk R, Somers VK. Influence of cardiac function and failure on sleep-disordered breathing: evidence for a causative role. $J$ Appl Physiol 2005;99:2433-9.

19. Giannoni A, Baruah R, Willson K, et al. Real-time dynamic carbon dioxide administration: a novel treatment strategy for stabilization of periodic breathing with potentialapplication to central sleep apnea. J Am Coll Cardiol 2010;56:1832-7.

20. Francis DP, Davies LC, Piepoli M, et al. Origin of oscillatory kinetics of respiratory gas exchange in chronic heart failure. Circulation 1999:100:1065-70.

21. Schmidt HB, Rauchhaus M, Francis DP, et al. Assessment of chemoreflex sensitivity in free breathing young subjects by correction for respiratory influence. Int J Cardiol 2001;78:157-65.

22. Davies LC, Colhoun $\mathrm{H}$, Coats AJ, et al. A non-invasive measure of baroreflex sensitivity without blood pressure measurement. Am Heart J 2002;143:441-7.

23. Penzel T, Mayer J, Peter JH, et al. Continuous non-invasive blood pressure monitoring in patients with sleep disorders. Physiol Res 1992;41:11-7.

24. Pryor WW. Cheyne-Stokes respiration in patients with cardiac enlargement and prolonged circulation time. Circulation 1951;4:233-8.

25. Crowell JW, Guyton AC, Moore JW. Basic oscillating mechanism of Cheyne-Stokes breathing. Am J Physiol 1956;187:395-8.

26. Tobin MJ, Snyder JV. Cheyne-Stokes respiration revisited: controversies and implications. Crit Care Med 1984;12:882-7.

27. Onal E, Burrows DL, Hart RH, et al. Induction of periodic breathing during sleep causes upper airway obstruction in humans. J Appl Physiol 1986;61:1438-43.

28. Szollosi I, Jones $\mathrm{M}$, Morrell $\mathrm{MJ}$, et al. Effect of $\mathrm{CO}_{2}$ inhalation on central sleep apnea and arousals from sleep. Respiration 2004;71:493-8.

29. Bradley TD, Takasaki Y, Orr D, et al. Sleep apnea in patients with left ventricular dysfunction: beneficial effects of nasal CPAP. Prog Clin Biol Res 1990;345:363-8. discussion 368-70. 